\section{Chrischta Ganz}

Seit jeher versucht der Mensch, die ihn umgebende Welt zu verstehen und zu erklären. Eine wichtige Grundlage für Antworten auf diese Fragen spielen spezifische Urprinzipien, aus denen sich alle Lebensprozesse ableiten lassen.

Sich dabei an der Natur zu orientieren, bringt die Elemente bzw. die Wahrnehmung ihrer Auswirkung auf Mensch und Natur in den Fokus des Weltverständnisses. In Europa und im arabischen Raum sind diese Urprinzipien als die vier Elemente Wasser, Erde, Luft und Feuer bekannt. In der chinesischen Tradition wird von den fünf Elementen Holz, Feuer, Erde, Metall und Wasser gesprochen.

Ihre Bedeutung ist nicht auf materielle Substanzen reduziert. Die Elemente stellen Lebensprinzipien und Qualitäten dar. Die Elementenlehre ist ein zentraler Baustein verschiedenster Lehren und bildet die philosophische Basis aller naturgemässen Heiltraditionen und Medizinalsysteme. Der Heilkundige kann auf dieser Grundlage die Diagnose individuell erkennen und die Therapie dementsprechend gestalten. Aristoteles (384322 v. Chr.) schreibt, dass alles, also auch der Mensch, aus den vier Elementen bestehe, jeweils individuell in anderer Zusammensetzung [1]. Ein solches naturphilosophisches Konzept formuliert auch Hildegard von Bingen (1098-1179). Die Elemente sind für sie die kosmischen Grundbausteine der Welt und all ihrer Geschöpfe. Der menschliche Körper ist aus Feuer, Luft, Wasser und Erde erschaffen. Dabei bildet das Feuer die Körperwärme und die Seele, die Luft trägt den Atem und das Gehör, die Erde nährt Körper, Knochen und Muskeln und das Wasser symbolisiert das Blut und die Bewegung (Abb. 1.).

\title{
Konstitutions- und Typenlehre
}

\section{Das Wesen der Elemente}

\section{Feuer}

Das Feuer steht für die ausstrahlende, anregende Energie, die Wärme und Licht hervorbringt. Feuer gilt als erhitzend und trocknend. Ein Übermass an Feuerkraft kann akute Entzündungen, Fieber, tachykarde Herzbeschwerden oder Bluthochdruck hervorrufen, ein Mangel hingegen Frösteln, Herzschwäche, niedrigen Blutdruck oder chronische Entzündungen auslösen.

\section{Luft}

Luft ist die Energie, die sich im Atem findet. Auch der Schlaf-/ Wachrhythmus und das Hormonsystem werden dem Element Luft zugeordnet. Im übertragenen Sinne steht Luft für Klarheit, Inspiration sowie Vernunft und wirkt anregend und befeuchtend. Zu viel Luft kann Krämpfe, zu wenig hingegen Lähmungserscheinungen hervorrufen.

\section{Wasser}

Die alles durchdringende Kraft des Wassers symbolisiert das lebenserhaltende Prinzip und wirkt kühlend und befeuchtend. Es wird mit Wachstum, Formgebung und Gefühl in Verbindung gebracht und den Körperflüssigkeiten zugeordnet. Ein Übermass an Wasser kann sich als Ödeme oder Verschleimung zeigen, ein Defizit ist beispielsweise als Trockenheit von Schleimhäuten und Haut spürbar.

\section{Erde}

Das Element Erde steht für die materielle, erstarrende, ruhende Energie und wird den kalt-trockenen Körperteilen wie Knochen und Gelenken zugeordnet. Ihre Qualität ist kühlend, austrocknend und zusammenziehend. Pathologische Abweichungen im Erdelement sind beispielsweise degenerative chronische Krankheiten wie Arthrose oder auch Depressionen.

\section{Bedeutung der Elemente für die Medizin}

Bei einem gesunden Menschen sind die Qualitäten der Elemente in einem ausgewogenen Verhältnis. Die individuelle Zusammensetzung macht den Charakter des Menschen aus. Durch Schwächung oder Mangel
Abb. 1. Im Alltag ist die Qualität der Elemente bekannt, beispielsweise in der Aussage «Ich bin nah am Wasser gebaut».

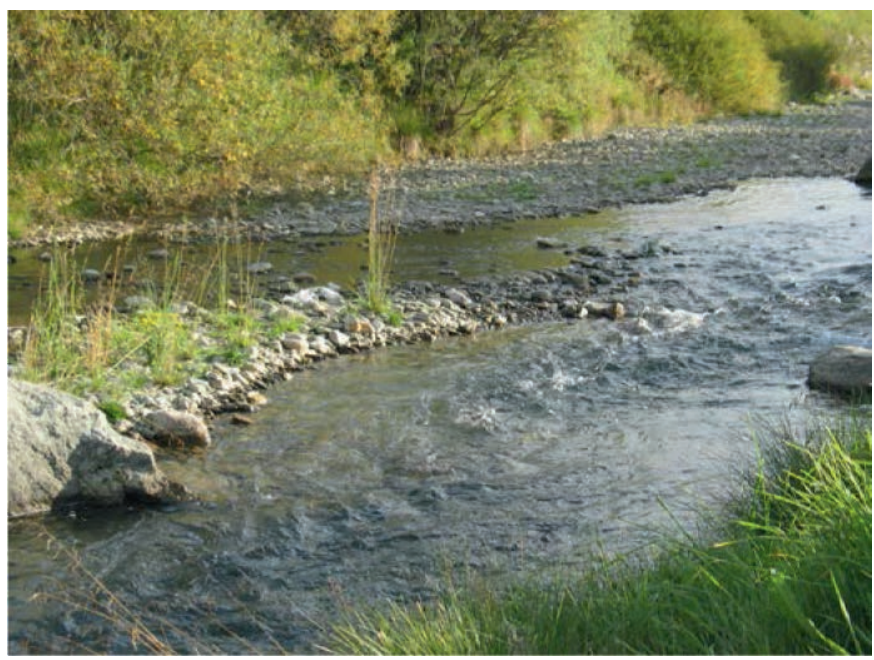

\section{KARGER}

Fax +4976145207 14
(๑) 2013 S. Karger GmbH, Freiburg
Chrischta Ganz

Praxis für Naturheilkunde

Steinentischstrasse 1, 8002 Zürich, Schweiz

naturheilpraxis@chrischtaganz.ch 
bzw. Überbetonung eines Elements entsteht Krankheit. Auch die Therapien und Heilmittel werden den Qualitäten der Elemente zugeordnet [2].

So kann Fieber (übermässiges Feuer) z.B. mit kühlender Weidenrinde (Salicis cortex) behandelt werden. Frösteln (zu viel Kälte) kann mit anregendem, wärmendem Rosmarin (Rosmarini folium) therapiert werden. Auf der europäischen Elementenlehre gründet auch die Temperamentenlehre mit den vier Charakteren Sanguiniker (Luft), Phlegmatiker (Wasser), Choleriker (Feuer) sowie Melancholiker (Erde). Es versteht sich von selbst, dass jeder Mensch eine Mischform darstellt.

Wie hier exemplarisch am Beispiel der europäischen Elementenlehre aufgezeigt, lässt sich das gleiche Denken auch in anderen Medizinalsystemen (Traditionelle Chinesische Medizin, Ayurveda, Traditionelle Tibetische Medizin usw.) aufzeigen.

\section{Konstitutionslehre}

Eine systemisch aufgebaute Medizin kann niemals davon ausgehen, dass der Husten von «Herr Meier» derselbe ist wie der Husten von «Frau Meier». Jedem Individuum liegen spezifische Reaktionsmuster, Eigenschaften und Merkmale zugrunde, sowohl auf physischer als auch auf seelischer und geistiger Ebene. Aufgrund dieser Informationen lassen sich Therapiekonzepte entwickeln, die nicht allein auf die aktuelle Krankheit fokussieren, sondern die den Organismus in seiner Ganzheit ausführlich erfassen und therapeutisch ebenso wie prophylaktisch stabilisieren [3].

Auf der Basis der vererbten Merkmale (Genotypus) entwickelt sich die Konstitution, die phänotypisch verändert - sowohl in stabilisierender als auch in destabilisierender Weise. Die konstitutionelle Gesamtsituation ist somit abhängig von der Lebensweise sowie von allen seelisch-geistigen, sozialen und ökologischen Be- dingungen [1]. Die aktuelle Lebenssituation sowie die Erkrankung und deren Heilungsmöglichkeiten werden vom spezifischen Muster eines Individuums, auf exogene und endogene Reize zu reagieren, sowie der Neigung zu bestimmten Erkrankungen und Fehlreaktionen (Disposition und Diathese) und von der aktuellen Lebenssituation geprägt.

Typologien helfen, charakteristische Reaktionsmuster im Krankheitsfall zu bestimmen und so dem Patienten die individuell bestmögliche Therapie zu ermöglichen. Die Unterscheidung nach den Elementen ist eine bewährte Möglichkeit; es gibt jedoch unzählige Typologien, von denen hier einige kurz vorgestellt werden sollen. Allen gemeinsam ist die Absicht, sich nicht alleine an einem Krankheitsnamen zu orientieren, sondern den Menschen in seiner Ganzheit zu erfassen. Es wird deutlich, dass aufgrund der Konstitution der pathophysiologische Hintergrund systemisch erfassbar wird.

- In der Psychologie und der Körperarbeit sind der denkorientierte, der bedürfnisorientierte, der belastungsorientierte, der leistungsorientierte und der kontrollorientierte Typ bekannt.

- Auch in der Homöopathie nimmt die Konstitutionstherapie eine wichtige Stellung ein. Das homöopathische Konstitutionsmittel stellt die Summe der körperlichen und psychischen Eigenschaften eines Menschen dar und bezieht sich direkt auf Person, Erscheinungsbild, Charakter sowie die typischen Erkrankungen.

- In der Irisdiagnostik gibt bereits die Augenfarbe eine grundsätzliche Information über die typischen Reaktionsmuster eines Menschen. Ein Mensch mit blauen Augen wird der lymphatischen Konstitution zugeordnet, was bedeutet, dass bei ihm das Lymphsystem bei jeder Krankheit besonders in Erscheinung tritt - sichtbar in der Anfälligkeit für akute und chronische Entzündungen der Lymphorgane wie Mandeln, Lymphknoten sowie Erkrankungen der Schleimhäute (Katarrhe) und der Haut (Ekzeme). Dies ist auch oft erkennbar als sogenannte kompensatorische Ausscheidungsprozesse über Haut und Schleimhaut. Therapeutisch leitet sich daraus die Notwendigkeit ab, bei lymphatischer Konstitution das gesamte Lymphsystem zu unterstützen, beispielsweise durch pflanzliche, homöopathische oder spagyrische Mittel, aber auch mittels Ausleitungsverfahren oder manueller Lymphdrainage.

Die hämatogene Konstitution (griech. Blut = «haima») steht für die warme und feuchte Qualität der Reaktionsmuster. Bei Krankheiten sind in erster Linie Organsysteme mit direkter Verbindung zum Blut betroffen (Blutgefässe arteriell/venös, Herz, Leber-GallenSystem, Schilddrüse und Milz). Ist das Wärmeprinzip übersteigert, treten hyperkinetische Syndrome auf; bei reduziertem Wärmeprinzip treten hingegen hypokinetische Syndrome auf.

Die biliäre Konstitution (lat. «bilis» $=$ Galle) steht für die hitzigen und trockenen Qualitäten der Reaktionsmuster. Bei Krankheit sind oft das Leber-Galle-System, der Verdauungstrakt sowie alle Arten der Muskulatur betroffen, meist in hyperkinetischer Qualität, in Form von Spasmen, Koliken und akuten Entzündungen.

- Dr. Eduard von Grauvogl (18111877) orientierte sich an den vier organischen Grundsubstanzen Sauerstoff, Wasserstoff, Stickstoff und Kohlenstoff. Aus den biochemischen Kenntnissen seiner Zeit entwickelte er seine Konstitutionslehre mit drei Stoffwechseltypen: die oxygenoide, die hydrogenoide und die carbo-nitrogenoide Konstitution.

- Die Konstitutionstypologie nach Dr. med. Bernhard Aschner (1889- 
1960) unterscheidet drei Grundkonstitutionen (lymphatische, hämatogene, dyskratische/gemischte Konstitution) mit Untertypen und Mischkonstitutionen.

- Die Konstitutionstypologie nach Ernst Kretschmer (1888-1964) beschreibt ebenfalls mehrere Typen: Der leptosome Typ ist hager, hat einen schlanken Körperbau, ist misstrauisch, empfindlich, pedantisch, scheu, aber auch feinfühlig. Der pyknische Typ hat einen gedrungenen Körperbau mit starkem Fettansatz am Rumpf, aber eher dünne Extremitäten. Sein Gesicht ist rund, er ist gemütlich und gesellig, aber auch anfällig für
Stimmungsschwankungen. Der athletische Typ ist stark, kräftig gebaut, langsam, beständig, aber auch ungewöhnlich reizbar. Hinzu kommt ein dysplastischer Typus mit auffallend unförmigem Körperbau.

- Die Konstitutionstypologie nach William Herbert Sheldon (18981977) orientiert sich an den drei Keimblättern als konstitutionsprägende Elemente. Sheldon beschreibt den endomorphen (Darm, Verdauungsorgane), den mesomorphen (Muskulatur, Ausscheidungsorgane, Bindegewebe) und den ektomorphen Typen (Haut, Sinnesorgane, Nervensystem).
- Heute sind moderne Modelle wie die Typologie nach Blutgruppen, nach Nervensystem (parasympathikoton/sympathikoton) und nach Stoffwechseltypen bekannt auch sie haben den Anspruch, systemisch und individuell zu sein.

\section{Literatur}

1 Raimann C, Ganz C, Garvelmann F, Bertschi-Stahl H-D, Fehr-Streule R: Grundlagen der Traditionellen Europäischen Naturheilkunde. Schiedlberg, Bacopa, 2012.

2 Banzhaf H: Der Mensch in seinen Elementen. München, Heinrich Hugendubel, 1993.

3 Bierbach E: Naturheilpraxis heute. München, Urban und Fischer, 2009. 